Aim of the study: Vascular endothelial growth factor (VEGF) is one of the parameters that has been studied in differential diagnosis of malignant fluids. This study is aimed at evaluate applicability of serum, fluid VEGF level and fluid to serum VEGF ratio in the diagnosis of malignant pleural mesothelioma (MPM).

Material and methods: The patients with pleural effusion over age of 18 , between 2011 and 2015 were included in the study. They were divided into three groups: group 1 - mesothelioma patients; group 2 - other malignancies; and group 3 - benign aetiologies. Group 1 and 2 were termed as the malignant group. Fluid, serum VEGF levels, and the ratio of fluid/serum VEGF level were studied to evaluate the flu$\mathrm{id} /$ serum VEGF ratio in all groups.

Results: Twenty cases with mesothelioma, 44 cases with other malignancies, and 20 cases with benign aetiologies were included in this study. No statistically significant difference was found according to serum VEGF levels for all groups, (group 1: $437 \pm 324 \mathrm{pg}$ / $\mathrm{ml}$, group 2: $354 \pm 223 \mathrm{pg} / \mathrm{ml}$, group 3: $373 \pm 217 \mathrm{pg} / \mathrm{ml}, p=0.836$ ), while fluid VEGF levels showed a statistically significant difference (group 1: $3359 \pm 700 \mathrm{pg} / \mathrm{ml}$, group 2: $2175 \pm 435$ $\mathrm{pg} / \mathrm{ml}$, group 3: $1092 \pm 435 \mathrm{pg} / \mathrm{ml}$, $p=0.041$ ). The ratio of fluid to serum VEGF levels showed a difference, at the significance limit, between the malignant (group 1 and group 2) and benign (group 3) groups ( $8.83 \pm 1.29$ vs. $4.57 \pm 1.07, p=0.059$ ) but showed a statistically significant difference between the mesothelioma and benign groups $(12.11 \pm 1.68$ vs. 4.57 $\pm 1.07, p=0.044$ ).

Conclusions: The VEGF fluid/serum ratio may be an applicable parameter in the differential diagnosis of malignant fluids, especially MPM.

Key words: pleural effusion, vascular endothelial growth factor (VEGF), malignant mesothelioma, pleural fluid/ serum VEGF ratio.

Contemp Oncol (Pozn) 2017; 21 (3): 213-217 DOI:https://doi.org/10.5114/wo.2017.70111

\section{The importance of serum and pleural fluid level of vascular endothelial growth factor (VEGF) and VEGF fluid/serum ratio in the differential diagnosis of malignant mesothelioma-related pleural effusion}

\author{
Yakup Düzköprü${ }^{1}$, Zeynep Oruç², Muhammet A. Kaplan², Refik Ülkü̈, \\ Çetin Tanrıkulu ${ }^{3}$, Derya Esmer ${ }^{1}$, Ali Bırak³ ${ }^{3}$ Mehmet Küçüköner ${ }^{2}$, \\ Zuhat Urakçı ${ }^{2}$ Abdurrahman Işıkdoğan ${ }^{2}$
}

${ }^{1}$ Department of Internal Medicine, Faculty of Medicine, Dicle University, Turkey 2Department of Medical Oncology, Faculty of Medicine, Dicle University, Turkey ${ }^{3}$ Department of Thoracic Surgery, Faculty of Medicine, Dicle University, Turkey ${ }^{4}$ Department of Chest Disease, Faculty of Medicine, Dicle University, Turkey

\section{Introduction}

Pleural effusion is a common clinical finding in malignant and benign diseases. In the differential diagnosis of pleural effusions, many causes of pleural effusion have been defined, to date. Malignant pleural effusion is one of the complications in cancer patients, which impairs the quality of life and it is often associated with a poor prognosis. The mean survival duration in malignant pleural effusion patients varies between four and nine months [1, 2]. In approximately $50 \%$ of cancer patients with a metastatic disease, malignant pleural effusion develops during the course of the disease [3]. There are many hypotheses about the pathogenesis of malignant pleural effusion. Pleural fluid accumulation associated with cancer can occur as a result of several factors, including mediastinal lymph node infiltration and pleural metastasis [1]. Recent studies have demonstrated that excessive production of vascular endothelial growth factor (VEGF) can play an important role in the pathogenesis of malignant pleural effusion [4, 5].

In the diagnosis of pleural effusion, clinical history, physical examination, imaging, and analysis of pleural fluid are usually sufficient to identify the underlying cause [6]. However, in some cases, it is difficult to differentiate benign from malignant pleural effusion, which makes the diagnosis of mesothelioma and lung adenocarcinoma. The specificity of cytological evaluation for pleural fluid is high, but the sensitivity is low. Several studies have reported that VEGF, which is high in malignancy-related pleural fluid, may be used for the differential diagnosis with other parameters [7].

Malignant pleural mesothelioma (MPM) is a rare and aggressive tumour, which arises from the pleural serous membrane surface. Most patients with MPM present with pleural effusion. Several studies have indicated that VEGF levels in pleural fluid of MPM patients are higher than those of patents with other malignant pleural effusions [8-10].

In addition, VEGF is a mediator with important functions in angiogenesis (vascular endothelial cell proliferation, differentiation, and tube formation). It plays an important role in malignant pleural effusion formation by increasing vascular permeability and vascular leakage, in addition to its important role in 
vascular development [4, 11-14]. In addition to mesothelial cells, tumour cells and infiltrating inflammatory cells in the pleural space all contribute to VEGF production [5].

Although several studies have been conducted about serum and pleural fluid levels of VEGF, the role of fluid/ serum VEGF ratio in the differentiation of mesothelioma-related pleural effusion has not been investigated, yet. Therefore, in the present study, we aimed to investigate the efficacy of the pleural fluid/serum VEGF ratio, in addition to VEGF levels of pleural fluid and serum, in the differentiation of malignant pleural mesothelioma from other malignant and benign effusions.

\section{Material and methods}

This study included patients over the age of 18 years, who had a diagnosis of pleural effusion with chest radiography and pleural fluid analysis at Dicle University Faculty of Medicine hospital between 2011 and 2015. This study was prospectively designed. All patients underwent diagnostic thoracentesis, and the pleural fluid analysis was examined. All patients' cytological evaluation and light's criteria including biochemical markers, serum, and pleural VEGF levels were examined. In patients with non-mesothelioma malignancy diagnosis, the cytological analysis of pleural fluid was examined. Eighty-four patients with pleural effusion were divided into three groups according to

Table 1. Distribution of 84 patients according to pleural effusion aetiology

\begin{tabular}{|lc|}
\hline Aetiology & Number of patients $n$ (\%) \\
\hline Benign $(n=20)$ & \\
\hline Heart failure & $7(35)$ \\
\hline Renal failure & $3(15)$ \\
\hline Pneumonia & $5(25)$ \\
\hline Liver Cirrhosis & $5(25)$ \\
\hline mesothelioma $(n=20)$ & \\
\hline Epithelial type & $14(70)$ \\
\hline Sarcomatoid type & $1(5)$ \\
\hline Biphasic (mix)type & $2(10)$ \\
\hline Unknown sub-type & $3(15)$ \\
\hline Non-mesothelioma & \\
\hline Malignancies $(n=44)$ & \\
\hline Lung cancer & $18(40.9)$ \\
\hline Non-Hodgkin's Lymphoma & $6(13.6)$ \\
\hline Breast cancer & $5(11.3)$ \\
\hline Over cancer & $2(4.5)$ \\
\hline Colon cancer & $4(9.1)$ \\
\hline Gastric cancer & $4(9.1)$ \\
\hline Other (pancreas, osteosarcoma, & $5(11.3)$ \\
\hline Bladder, prostate, larynx cancer) & \\
\hline
\end{tabular}

aetiologies. Group 1 included patients with a diagnosis of mesothelioma (histologically confirmed); group 2 included patients with non-mesothelioma malignancies (histologically confirmed) and whose pleural fluid cytological analysis was cytology-positive; and group 3 included patients whose pleural effusion was related to non-malignant disease and had normal pleural effusion cytology analysis. Pleural fluid and serum samples were collected. Patients on medical treatments such as anti-inflammatory drugs or corticosteroids and anticancer therapy were excluded from the study.

This study was approved by the Ethics Committee of Dicle University and conducted in accordance with the principles of the Declaration of Helsinki. Written, informed consent was obtained from each patient. Before therapy 10 cc peripheral venous blood samples and 10 cc pleural effusion samples were taken via thoracentesis. Serum was separated from the blood samples, which were centrifuged at $4000 \mathrm{rpm}$ for $10 \mathrm{~min}$, and samples were stored at $-80^{\circ} \mathrm{C}$ until analysis. The pleural fluid and serum samples, which were prepared, centrifuged, and held at $-80^{\circ} \mathrm{C}$ until analysis, were analysed after reaching room temperature. The VEGF levels were measured with the enzyme-linked immunosorbent assay (ELISA) method using an eBioscience kit.

\section{Statistical analysis}

Statistical analysis was performed using SPSS version 17 software (SPSS Inc., Chicago, IL, USA). When comparing the two groups, the Student t-test, Mann-Whitney U-test, Kruskal-Wallis test, and analysis of variance (ANOVA) test were used for numerical parameters. The $\chi^{2}$ and Fisher's exact tests were used for categorical parameters. The cutoff value for fluid/serum VEGF ratio between malignant and benign groups was calculated with receiver operating characteristics (ROC) analysis. A p value of less than 0.05 was considered statistically significant.

\section{Results}

Eighty-four patients were included in the study: 20 patients with mesothelioma constituted group 1, 44 patients with non-mesothelioma constituted group 2, and 20 patients with benign pleural effusion constituted group 3 (Table 1). Among the patients with mesothelioma diagnosis, 14 (70\%) were epithelial, one patient was (5\%) sarcomatoid, two were (10\%) biphasic (mixed), and the sub-type of three patients (15\%) was unknown. The distribution of patients with non-mesothelioma malignancy in group 2 was as follows: $40.9 \%(n=18)$ lung carcinoma, $13.6 \%(n=6)$ non-Hodgkin's lymphoma, $11.3 \%(n=$ 5) breast carcinoma, 9.1\% $(n=4)$ colon carcinoma, $9.1 \%$ $(n=4)$ gastric carcinoma, $4.5 \%(n=2)$ over carcinoma, and $11.3 \%(n=5)$ other (pancreas, bladder, carcinoma, osteosarcoma, prostate carcinoma, and larynx carcinoma). Group 3 with benign pleural effusion was composed of $25 \%(n=5)$ pneumonia, 25\% $(n=5)$ liver cirrhosis, 35\% $(n=7)$ heart failure, and $15 \%(n=3)$ acute renal failure (Table 1$)$.

The demographic characteristics of the patients were as follows: the median age of patients in group 1 was 55 years (range: 22-74); in group 2 was 57.5 years (range: 
Table 2. Age and gender distribution of groups and results of serum/pleural fluid analysis

\begin{tabular}{|c|c|c|c|c|}
\hline & $\begin{array}{c}\text { Group } 1 \\
\text { (mesothelioma) } \\
(n=20)\end{array}$ & $\begin{array}{c}\text { Group } 2 \\
\text { (non-mesothelioma } \\
\text { malignancy) }(n=44)\end{array}$ & $\begin{array}{l}\text { Group } 3 \text { (benign) } \\
\quad(n=20)\end{array}$ & $p$ value \\
\hline Age & $55(22-74)$ & $57(27-81)$ & $66(19-82)$ & 0.482 \\
\hline Gender & $\begin{array}{l}\mathrm{E}(n=11 ; 55 \%) \\
\mathrm{K}(n=9 ; 45 \%)\end{array}$ & $\begin{array}{l}\mathrm{E}(n=26 ; 59.1 \%) \\
\mathrm{K}(n=18 ; 40.9 \%)\end{array}$ & $\begin{array}{l}\mathrm{E}(n=11 ; 55 \%) \\
\mathrm{K}(n=9 ; 45 \%)\end{array}$ & 0.931 \\
\hline \multicolumn{5}{|l|}{$\begin{array}{l}\text { Pleural fluid (light criteria) } \\
\text { (mean } \pm \text { SD) }\end{array}$} \\
\hline T. protein & $3.97 \pm 1.17$ & $3.25 \pm 0.83$ & $2.85 \pm 1.5$ & $\begin{array}{l}0.012 \\
\text { Group 1 - Group 2: } 0.13 \\
\text { Group 1 - Group 3: } 0.08 \\
\text { Group 2-Group 3: } 0.32\end{array}$ \\
\hline Albumin & $1.78 \pm 0.80$ & $1.52 \pm 0.51$ & $1.23 \pm 0.58$ & 0.29 \\
\hline Glucose & $75 \pm 27.4$ & $107 \pm 60.3$ & $103 \pm 38.5$ & 0.97 \\
\hline LDH & $549 \pm 437$ & $615 \pm 613$ & $539 \pm 621$ & 0.85 \\
\hline VEGF fluid (mean \pm SD) & $3359 \pm 700$ & $2175 \pm 435$ & $1092 \pm 435$ & $\begin{array}{l}\text { O.04 } \\
\text { Group 1 - Group 2: } 0.23 \\
\text { Group 1-Group 3: } 0.01 \\
\text { Group 2-Group 3: } 0.08\end{array}$ \\
\hline VEGF serum $($ mean $\pm S D)$ & $437 \pm 324$ & $354 \pm 223$ & $373 \pm 217$ & 0.836 \\
\hline $\begin{array}{l}\text { VEGF fluid/serum ratio } \\
\text { (mean } \pm \text { SD) }\end{array}$ & $12.11 \pm 1.68$ & $7.19 \pm 1.04$ & $4.57 \pm 1.07$ & $\begin{array}{c}0.10 \\
\text { Group 1 - Group 2: } 0.822 \\
\text { Group 1-Group 3: } 0.044 \\
\text { Group (1+ 2) - Group 3: } \\
0.059\end{array}$ \\
\hline
\end{tabular}

27-81); and in group 3 was 66.5 years (range: 19-82) $(p=0.48)$. Of 84 patients, $48(57 \%)$ were male and $36(43 \%)$ were female. There was no statistically significant difference among the groups in terms of age and sex (Table 2).

According to the pleural fluid analysis, the mean total protein level was $3.97 \pm 1.17 \mathrm{~g} / \mathrm{dl}$ in group $1,3.25 \pm 0.83$ $\mathrm{g} / \mathrm{dl}$ in group 2 , and $2.85 \pm 1.5 \mathrm{~g} / \mathrm{dl}$ in group 3 ( $p=0.012$ ) In the sub-group analysis, the differences between group 1 and group $2(p=0.137)$ and between group 2 and group 3 $(p=0.327)$ were not statistically significant. The difference between group 1 and group 3 was statistically significant ( $p=0.008)$. The mean albumin level was $1.78 \pm 0.8 \mathrm{~g} / \mathrm{dl}$ in group 1, $1.52 \pm 0.51 \mathrm{~g} / \mathrm{dl}$ in Group 2, and $1.23 \pm 0.58 \mathrm{~g} / \mathrm{dl}$ in group $3(p=0.295)$. The mean glucose level was $75 \pm 27.4$ $\mathrm{g} / \mathrm{dl}$ in Group 1, $107 \pm 60 \mathrm{~g} / \mathrm{dl}$ in group 2, and $103 \pm 38,5 \mathrm{~g} / \mathrm{dl}$ in group $3(p=0.97)$. The mean LDH level was $549 \pm 437$ in Group 1, $615 \pm 613$ in group 2, and $539 \pm 621$ in group 3. The differences between groups were not statistically significant ( $p=0.857$ ) (Table 2).

The mean VEGF level in pleural effusion was detected as $3359 \pm 700 \mathrm{pg} / \mathrm{ml}$ in group 1, $2175 \pm 435 \mathrm{pg} / \mathrm{ml}$ in group 2, and $1092 \pm 435 \mathrm{pg} / \mathrm{ml}$ in group $3(p=0.041)$ in the sub-group analysis, while the difference between group 1 and group 2 $(p=0.233)$ and between group 2 and group $3(p=0.086)$ was not statistically significant. The difference between group 1 and group 3 was statistically significant ( $p=0.014$ ). The mean serum VEGF level was $437 \pm 324 \mathrm{pg} / \mathrm{ml}$ in group 1 , $354 \pm 223 \mathrm{pg} / \mathrm{ml}$ in group 2, and $373 \pm 217 \mathrm{pg} / \mathrm{ml}$ in group 3 $(p=0.836)$. Serum and pleural VEGF levels of the groups are shown in Fig. 1.

\section{Fluid/serum VEGF ratio}

In our study, the mean fluid/serum VEGF ratio in all malignant patients was $8.83 \pm 1.29$. In the sub-group analysis, the fluid/serum VEGF ratio was $12.11 \pm 1.68$ in the mesothelioma group, $7.19 \pm 1.04$ in the non-mesothelioma malignancy group, and $4.57 \pm 1.07$ in the benign group ( $p=0.10$ ) The ratio of fluid to serum VEGF levels showed a difference close to the level of statistical significance between malig-

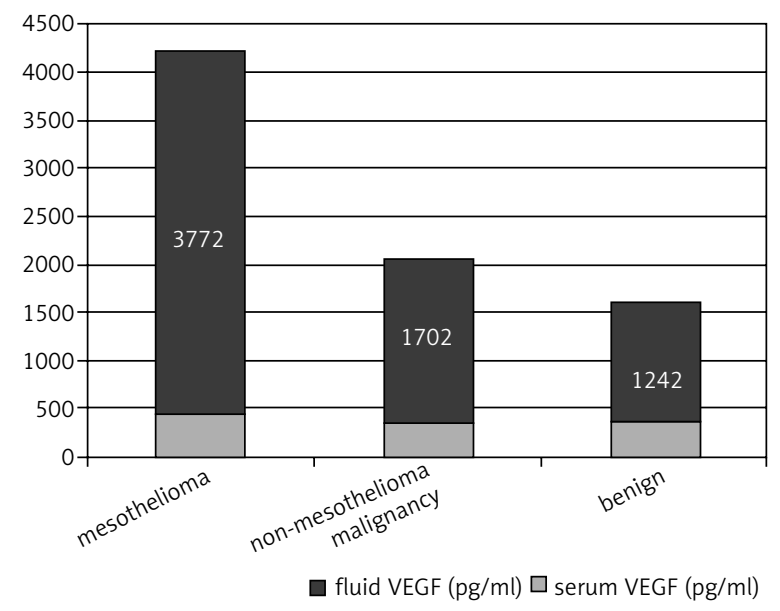

Fig. 1. Serum and pleural VEGF levels of the groups 
nant (group 1 and group 2) and benign (group 3) groups (8.83 \pm 1.29 vs. $4.57 \pm 1.07, p=0.059)$. The difference between the mesothelioma and benign groups was statistically significant ( $p=0.044)$ (Table 2$)$.

According to the ROC analysis, the fluid/serum VEGF ratio was detected as 0.85 , the cut-off value, with $76 \%$ sensitivity and $50 \%$ specificity. According to the cut-off value, patients were categorised as under 0.85 and over 0.85 . According to this, the ratio of patients whose fluid/serum VEGF ratio was under 0.85 was $24.1 \%$ in the malignant group, $50 \%$ in the benign group, $22.2 \%$ in the mesothelioma group, and $25 \%$ in the non-mesothelioma malignancy group. In the sub-group analysis, the difference between the malignant group and the benign group was statistically significant ( $p=0.032$ ), and the value between the mesothelioma and benign groups was at the significance limit $(p=0.076)$.

\section{Discussion}

Only $50 \%$ of pleural effusion cases in cancer patients are malignant pleural effusion [5]. The most specific non-invasive technique for the aetiology in malignant pleural effusion is cytological evaluation of pleural fluid. However, the sensitivity of cytological evaluation in malignancies is only $60 \%$. In cases of mesothelioma, this ratio decreases to $10 \%[6,15]$. Thus, invasive diagnostic procedures, such as pleural biopsy or thoracoscopic bronchoscopy, can be required for histopathological evaluation [16].

Recently, new molecular markers were investigated to increase the diagnostic value of pleural fluid analysis. To date, many biochemical markers including tumour markers have been studied in malignant pleural effusion, and the difference in marker levels was detected between malignant and benign effusions; however, none of them showed sufficient sensitivity and specificity.

High VEGF levels were shown both in malignant and benign pleural effusions [13]. This is thought to be related to expression of VEGF by normal tissues such as lung, kidney, heart, liver, adrenal glands, gastric mucosa, and immunity cells, in addition to cancer cells in malignancies [12].

A significant difference was detected between benign and malignant pleural effusions in terms of VEGF and there were higher VEGF values in malignant pleural effusions [7, 17-19]. In a meta-analysis that evaluated the diagnostic value of VEGF, the sensitivity of pleural fluid VEGF was 75\% (95\% Cl: 0.72-0.79) and specificity was 72\% (95\% $\mathrm{Cl}$ : 0.68-0.76). According to the results of the meta-analysis, VEGF can be used in the diagnosis of malignant pleural effusion, but is not sufficient to verify a malignant pleural effusion diagnosis because the specificity of VEGF is very low. The authors recommended that VEGF should be evaluated with other pleural effusion parameters or other markers in the diagnosis of malignant pleural effusion [12]. In the study of Zhou et al., the diagnostic value of VEGF and endostatin in the differential diagnosis of malignant and tuberculous pleural effusion was investigated. Because each of them have low sensitivity and specificity, they are not useful alone in the diagnosis of malignant pleural effusion; however, the study recommended that when VEGF and endostatin were evaluated together, they could be used in the differential diagnosis as sensitivity and specificity increases (81\% and 97\%, respectively) [18]. Furthermore, in a study of Fiorelli et al., the concomitant use of VEGF and cytological analysis increased the probability of detection of malignancy by $24 \%$ [17].

VEGF and angiogenesis play an important role in the pathophysiology and progression of mesothelioma [20]. In a study of Fiorelli et al., pleural fluid VEGF levels were compared between malignant diseases (lung cancer $n=14$, mesothelioma $n=13$, extra thoracic malignancies $n=25$ ). VEGF levels were not different between lung cancer and other malignant disease (extra thoracic cancer and mesothelioma)-related effusions [17]. On the other hand, in our study, patients with mesothelioma had higher VEGF levels in malignant pleural effusions when compared with other cancer patients.

Although various studies have been conducted on serum and pleural fluid levels of VEGF, the role of the fluid/ serum VEGF ratio in the distinction of malignant-benign effusions was only evaluated in one study. In the study of Sack et al., the differences in the VEGF pleural fluid/serum ratio were detected between malignancies (lung cancer and secondary malignancies with pleural metastasis) and other benign diseases. In the malignant group, pleural fluid/serum VEGF ratios were generally over 1, although they could be different from each other. In benign diseases related to infections and tuberculosis, this ratio was over 1 ; in heart failure, this ratio was under 1 [7]. To date, there have been no studies investigating the VEGF pleural fluid/ serum ratio in mesothelioma patients.

Furthermore, in our study, both serum and pleural fluid VEGF levels and the pleural fluid/serum ratio were investigated in mesothelioma patients. A difference at the significance limit was detected between all malignancies (mesothelioma and non-mesothelioma malignancies) and in the benign group in terms of the pleural fluid/serum VEGF ratio ( $p=0.059)$. In the sub-group analysis, the highest ratio was found in the mesothelioma group, and the lowest ratio was detected in the benign group. The difference between the mesothelioma group and the benign group was statistically significant $(p=0.044)$. When patients were categorised according to the cut-off value of 0.85 , the difference between all malignancies and the benign group was statistically significant ( $p=0.032$ ). In the sub-group analysis, the difference between the mesothelioma and benign groups was at the significance limit $(p=0.076)$. In our study, the fluid/serum VEGF ratio sensitivity was $76 \%$ and specificity was 50\%. No statistically significant difference was detected between the non-mesothelioma malignant group and the benign group $(p=0.822)$ and between the mesothelioma group and the non-mesothelioma malignant group ( $p=0.279$ ).

Previous studies have shown that pleural fluid VEGF levels in malignant pleural effusions are higher than serum levels, but there is no correlation between pleural fluid and serum levels [5]. In our study, pleural fluid VEGF levels in all three groups were higher than serum levels, and there was no statistically significant difference between the groups in terms of serum VEGF levels $(p=0.836)$. 
When other studies were evaluated, the hypothesis was supported that in malignant pleural effusions VEGF is generally released from malignant cells locally in the pleural space. Therefore, we can say that studies that chiefly evaluate pleural fluid VEGF levels can produce more accurate results. Based on these results, it should be kept in mind that the pleural amount of viable tumour burden in malignant diseases can affect VEGF levels, and each cancer may have different ratios of VEGF expression. Although there was a numerical difference in our study, some ratios and data at significance limit might be due to the small sample size. More accurate results can be obtained with larger patient groups. Our study includes some limitations such as a limited number of cases and the fact that the benign group was heterogeneous.

The VEGF and pleural fluid/serum VEGF ratio can be used in malignant pleural effusion and malignant pleural mesothelioma as a diagnostic marker and for choosing appropriate patients who can benefit from more invasive procedures.

In conclusion, VEGF can be a useful parameter in the differential diagnosis of malignant pleural fluids, particularly malignant pleural mesothelioma. Further studies are needed which can support the applicability of fluid/serum VEGF ratio as a promising marker in differential diagnosis of malignant fluids. However, these parameters should be evaluated with other parameters that have been used in the diagnosis of different malignant pleural fluids.

The authors declare no conflict of interest.

\section{References}

1. Uzbeck MH, Almeida FA, Sarkiss MG. Management of Malignant Pleural Effusions. Adv Ther 2010; 27: 334-347.

2. Bielsa S, Martin-Juan J, Porcel JM, Rodríguez-Panadero F.Diagnostic and prognostic implications of pleural adhesions in malignant effusions. J Thorac Oncol 2008; 3: 1251-6.

3. Heffner JE, Klein JS. Recent advances in the diagnosis and management of malignant pleural effusions. Mayo Clin Proc 2008; 83: 235-50.

4. Brown LF, Detmar M, Claffey K Brown LF, Detmar M, Claffey K, et al. Vascular permeability factor/vascular endothelial growth factor: a multifunctional angiogenic cytokine. EXS 1997; 79: 233-69.

5. Bradshaw M, Mansfield A, Peikert T. The role of vascular endothelial growth factor in the pathogenesis, diagnosis and treatment of malignant pleural effusion. Curr Oncol Rep 2013; 15: 207-216.

6. Porcel MJ. Pearls and myths in pleural fluid analysis. Respirology 2011; 16: 44-52.

7. Sack U, Hoffmann M, Zhao XJ. Vascular endothelial growth factor in pleural effusions of different origin. Eur Respir J 2005; 25: 600-4.

8. Ohta Y, Shridhar V, Bright RK. VEGF and VEGF type C play an important role in angiogenesis and lymphangiogenesis in human malignant mesothelioma tumours. Br J Cancer 1999; 81: 54-61.

9. Yasumitsu A, Tabata C, Tabata R. Clinical significance of serum vascular endothelial growth factor in malignant pleural mesothelioma. J Thorac Oncol 2010; 5: 479-83.

10. Hirayama N, Tabata C, Tabata R, et al. Pleural effusion VEGF levels as a prognostic factor of malignant pleural mesothelioma. Respir Med 2011; 105: 137-42.

11. Hicklin DJ, Ellis LM. Role of the vascular endothelial growth factor pathway in tumor growth and angiogenesis. I Clin Oncol 2005; 23: 1011-27.
12. Shen Y, LIU M, Wan C. Diagnostic accuracy of vascular endothelial growth factor for malignant pleural effusion: A meta-analysis. Exp Ther Med 2012; 3: 1072-6.

13. Grove CS, Lee YC. Vascular endothelial growth factor: the key mediator in pleural effusion formation. Curr Opin Pulm Med 2002; 8: 294-301.

14. Ishii H, Yazawa T, Sato H, et al. Enhancement of pleural dissemination and lymph node metastasis of intrathoracic lung cancer cells by vascular endothelial growth factors (VEGFs). Lung Cancer 2004; 45: 325-37.

15. Bhatnagar R. The modern diagnosis and managment of pleural effusions. BMJ 2015; 351: h4520.

16. Husain AN, Colby T, Ordonez N. Guidelines for pathologic diagnosis of malignant mesothelioma: 2012 update of the consensus statement from the International Mesothelioma Interest Group. Arch Pathol Lab Med 2013; 137: 647-67.

17. Fiorelli A, Vicidomini G, Di Domenico M, Napolitano F, Messina G, Morgillo F, Ciardiello F, Santini M. Vascular endothelial growth factor in pleural fluid for differential diagnosis of benign and malignant origin and its clinical applications. Interact Cardiovasc Thorac Surg 2011; 12: 420-4.

18.Zhou W-B, Bai M, Jin Y. Diagnostic value of vascular endothelial growth factor and endostatin in malignant pleural effusions. Int J Tuberc Lung Dis 2009; 13: 381-6.

19. Gu Y, Zhang M, Li G, et al. Diagnostic values of vascular endothelial growth factor and epidermal growth factor receptor for benign and malignant hydrothorax. Chin Med J (Engl) 2015; 128: 305-9.

20. Strizzi L, Catalano A, Vianale G. Vascular endothelial growth factor is an autocrine growth factor in human malignant mesothelioma. J Pathol 2001; 193: 468-75.

\section{Address for correspondence}

\section{Zeynep Oruç}

Department of Medical Oncology

Dicle University

21280 Diyarbakır, Turkey

e-mail: zeynep44oruc@hotmail.com

Submitted: 27.03 .2017

Accepted: $\quad 8.06 .2017$ 